\title{
The neural correlates of attempting to suppress negative versus neutral memories
}

\author{
ANDReW J. Butler ANd KARIN H. JAMeS \\ Indiana University, Bloomington, Indiana
}

\begin{abstract}
We performed an event-related fMRI study comparing attempts at suppressing recall of negative versus neutral memories. The hippocampus is crucial for successful explicit recall. Hippocampal activation has been shown to decrease during the suppression of previously learned neutral words. However, different effects may occur in the case of emotional memories. Participants first learned 40 word pairs consisting of a cue and either a neutral or a negative target. During fMRI scanning, the participants were shown the cues and were instructed to recall the targets or to suppress the targets, using attentional distraction. Similar right-lateralized frontoparietal regions were activated more during suppression than during recall, regardless of emotion. However, we show for the first time that lowered hippocampal activation occurs during the suppression of neutral, but not negative, words. Coinciding with this sustained hippocampal activation, the amygdala, insula, anterior cingulate, and fusiform gyrus showed greater activation during the suppression of negative memories than during suppression of neutral memories. Thus, during attempts to suppress negative memories, regions involved in the emotional and sensory aspects of memory reactivate, along with regions indexing conscious recall. Revealing the neural correlates and mechanisms of the suppression of negative memories has relevance for disorders such as posttraumatic stress disorder, in which traumatic memories often intrude and are associated with avoidance. Supplemental materials for this article may be downloaded from http://cabn.psychonomic-journals.org/content/supplemental.
\end{abstract}

Anecdotal reports and empirical evidence have suggested that emotion enhances the subjective sense of remembering and, to a lesser degree, the accuracy of memory (Phelps \& Sharot, 2008). From an evolutionary perspective, this is beneficial. Enhancement may lead to increased survival via quicker and more accurate responses to previously encountered stimuli that elicit emotional responses representing significance. However, as can be seen in such disorders as posttraumatic stress disorder (PTSD), the enhancement and persistence of memory for highly arousing and negative traumatic events can lead to devastating issues long after the event itself occurred (American Psychiatric Association, 2000). One way in which people cope with unwanted memories is by attempting to suppress them. Suppression of a memory is a construct that can be separated from physical avoidance of memory cues. It has been suggested that physical avoidance of memory cues may lead to a reinstatement of negative memories when a cue becomes unavoidable at a later time but that suppression of cued memories may allow for regulatory processes to occur, decreasing this reinstatement (Anderson, 2001).

There is some evidence suggesting that attempting to suppress recall may lead to poor emotional, behavioral, and cognitive outcomes (Dalgleish, Hauer, \& Kuyken, 2008; Ehlers \& Clark, 2000). Successful treatment of patients with traumatic memories has often involved the use of strategies that bring the memory into awareness in the ab- sence of the negative emotional reaction (Harvey, Bryant, $\&$ Tarrier, 2003). These interventions generally have not involved suppression. It has been argued that the therapeutic effects of evoking the experience of emotional memories may depend on a reappraisal of the prior experience (Littrell, 1998). Thought suppression research has demonstrated that some of the negative consequences of suppression are related to psychopathologies (Purdon, 1999). Naturalistic studies of suppression after traumatic events have shown that suppression is positively correlated with a greater degree of reexperience and posttraumatic stress (Joseph et al., 1996; Mayou, Ehlers, \& Bryant, 2002). Positive relationships between greater suppression of unwanted thoughts and greater nonsuicidal self-injury, suicidal ideation, and suicide attempts have been demonstrated (Najmi, Wegner, \& Nock, 2007). Controlled laboratory experiments of the suppression of traumatic negative memories have also suggested that these attempts may be unhelpful and, when successful, can even lead to a later reinstatement of the negative memories (Beck, Gudmundsdottir, Palyo, Miller, \& Grant, 2006; Dalgleish \& Yiend, 2006; Harvey \& Bryant, 1998; Shipherd \& Beck, 1999). Greater arousal has been detected during suppression, relative to the recall of arousing thoughts (Wegner, Shortt, Blake, \& Page, 1990). Suppression may serve to continue the vicious cycle of negative outcomes related to traumatic memories.

However, there is another, equally important view to consider. Some researchers have asserted that the causal 
influence of suppression in maintaining disorders, such as PTSD, has not been clearly established (Rassin, Merckelbach, \& Muris, 2000). It is possible that individuals with greater clinical problems might try harder to suppress unwanted thoughts and memories. In this case, suppression would be correlated with worse outcomes as a result of the clinical problem itself. Furthermore, a quantitative metaanalysis of thought suppression studies showed only a moderate increase in the intrusion of suppressed thoughts (Abramowitz, Tolin, \& Street, 2001). Some research has explicitly tested the possibility that suppression has beneficial effects. Dunn, Billotti, Murphy, and Dalgleish (2009) investigated whether emotion suppression versus acceptance would lead to different outcomes after highly negative videos were viewed. They found that, whereas emotion suppression leads to a decrease of negative emotion and memory over time, acceptance encouraged ongoing negative reactions to the experience. New research may overturn the commonly accepted view that suppression is entirely maladaptive or, at least, give a more balanced understanding of both its costs and its benefits.

Investigation of the mechanisms underlying the suppression of negative thoughts is one crucial step in resolving these issues and may help to optimize treatment in the future. We are only beginning to explore the neural processes and mechanisms underlying the suppression of negative versus neutral material in memory. A better understanding of the crucial mechanisms of suppression is now available to us through the use of new experimental paradigms that can be used in concert with neuroimaging techniques.

The think/no-think (TNT) paradigm allows one to study the effects of suppression on subsequent memory retrieval (Anderson \& Green, 2001). The TNT paradigm, in its original form, consists of three main phases. First, word pairs are learned. Second, the first word (cue) in the pair is presented with instructions to either think or not think of the second word of the pair (target). This is followed by a memory test where successful suppression is evidenced by the negative control effect, referring to when no-think targets are recalled at a significantly lower percentage, relative to baseline targets not presented during the second phase (Levy \& Anderson, 2008). Results from several published TNT studies have demonstrated this negative control effect, providing evidence that successful suppression can occur (Anderson \& Green, 2001; Anderson et al., 2004; Bergstrom, Velmans, Fockert, \& RichardsonKlavehn, 2007; Depue, Banich, \& Curran, 2006; Depue, Curran, \& Banich, 2007; Hanslmayr, Leipold, Pastötter, \& Bäuml, 2009; Hertel \& Calcaterra, 2005; Hertel \& Gerstle, 2003; Joorman, Hertel, Brozovich, \& Gotlib, 2005; Levy \& Anderson, 2008; Paz-Alonso, Ghetti, Matlen, Anderson, \& Bunge, 2009; Wessel, Wetzels, Jelicic, \& Merckelbach, 2005; but for an absence of the finding, see also Bulevich, Roediger, Balota, \& Butler, 2006; Mecklinger, Parra, \& Waldhauser, 2009). For a more detailed and recent overview of these issues, see Anderson and Levy (2009).

Anderson et al. (2004), in a replication of the original TNT behavioral study, included functional magnetic resonance imaging (fMRI) during the critical TNT phase. This allowed for the comparison of neural activity during recall versus suppression. Suppression of memories in the no-think task corresponded to the activation of areas constituting a frontoparietal network that has previously been found to be involved in executive control and inhibition of responses (Miller, 2000). Suppression, leading to later forgetting, was associated with the activation of the dorsal lateral prefrontal cortex, which has also been shown to activate during inhibition in the similar go/no-go task (Watanabe et al., 2002). Activation in the hippocampus was found to have decreased in no-think suppression trials, as compared with the think or retrieve trials. These results were interpreted as demonstrating active inhibition of conscious recall, as reflected in the downregulation of the hippocampus via executive inhibitory control exerted by the frontoparietal network (Anderson et al., 2004). Importantly for this claim, the results of another study, discussed below, indicated that activation in the hippocampus during suppression was below a passive fixation baseline (Depue et al., 2007). This suggests that the interpretation of hippocampal modulation reflecting a down-regulation relies on more than a relative difference between the think and no-think conditions. ERP studies of the TNT paradigm have provided further evidence supporting the claim that the avoidance of conscious recollection occurs during the suppression phase of this paradigm (Bergstrom et al., 2007; Hanslmayr et al., 2009; Mecklinger et al., 2009).

Considering the role of emotion in intentional forgetting is important for connecting the work discussed thus far to clinical concerns. Some evidence exists that emotional memories are harder to intentionally forget than neutral memories. For example, results from the list method of directed forgetting generally showed inhibition of the later recall of items from a forget list, relative to a remember list (Bjork \& Woodward, 1973). Extending this finding to emotional stimuli, Payne and Corrigan (2007) found that forget lists with emotional stimuli did not show the forgetting effect, whereas the lists of neutral stimuli did. Moderately high arousing images of both negative and positive valence showed lower forgetting rates than did neutral images of low arousal and intermediate valence. However, two studies looking at directed forgetting in autobiographical memories showed generally comparable directed forgetting effects when neutral memories were compared with emotional memories (Barnier et al., 2007; Joslyn \& Oakes, 2005). Differences in task requirements between directed forgetting and the TNT paradigm should be considered when results are compared. During the list method of directed forgetting, the instruction to forget comes once after the presentation of multiple stimuli, but in the TNT paradigm, there are many more instructed attempts to forget individual items (Levy \& Anderson, 2008).

Some work has investigated the effect of emotional variables on performance in the TNT paradigm. A study employing the TNT paradigm, using both words and images of negative or neutral valence, both with moderate arousal, showed that emotional no-think items tended to 
be forgotten to a greater extent than neutral items (Depue et al., 2006). A study using the TNT paradigm manipulated the level of arousal, as well as valence, using word pair stimuli (Marx, Marshall, \& Castro, 2008). Their results demonstrated that across a number of memory tests, unlike pleasant and less arousing negative words, highly negative words did not show a difference when think items were compared with no-think items. These results suggest the possibility that relatively highly arousing negative stimuli may not be as readily suppressed.

A recent TNT fMRI study (Depue et al., 2007) used negative images in the moderately arousing category of the stimuli set and showed that the suppression of these items initially involved the activation of the right inferior frontal gyrus, with an associated decrease in activation in regions implicated in the sensory representations of memories, including the visual cortex. Over repeated blocks in the TNT phase, the initial activation was followed by activation of the right medial frontal gyrus, with an associated decrease in the activation of other putative memory and emotional systems, including the hippocampus and amygdala. It is interesting to note that the control regions found in Depue et al. (2007) were similar to those found in Anderson et al. (2004), so there is reason to think they may be co-localized for both neutral and negative material. This study suggested mechanisms that might be involved when individuals successfully suppress emotional material in memory. However, the neural systems engaged during the suppression of negative stimuli in the TNT paradigm may differ from those engaged during suppression of neutral stimuli. This difference may reveal important information about what makes emotional memories persistent.

The main goal of the present study was to compare suppression of negative versus neutral stimuli directly, measuring neural activation patterns with fMRI. To this end, we used a modified version of the TNT, controlling for strategy across target type, to measure differences in neural activation during the critical TNT phase. The present study focused on a manipulation in which relatively highly arousing negative words versus low-arousing neutral words were used. In this way, we hoped to increase the understanding of what differences occur in neural activity during attempts to suppress negative versus neutral memories. We expected to replicate the results in Anderson et al. (2004) in which a relative decrease in hippocampal response during the suppression of neutral targets was shown. However, we expected that the hippocampal response would not decrease during attempts to suppress negative targets. A similar activation of a frontoparietal network was expected during the suppression of both neutral and negative words.

\section{METHOD}

\section{Participants}

Fifteen participants, with 1 excluded due to failure to learn the words, took part in the study. Seven participants were female, and 7 were male (mean age $=22.64$ years, $S D=3.88$ ). All the participants gave informed consent according to the guidelines of the Indiana University institutional review board. All the participants were right-handed, reported normal or corrected-to-normal vision, and had no known neurological damage. The participants were compensated for their time.

\section{Stimuli}

All the stimuli were chosen from the Affective Norms of English Words (ANEW; Bradley \& Lang, 1999). Each of the 40 word pairs consisted of a cue and a target. All 40 cues were neutral (low arousal with intermediate valence). Half of the target words were neutral (low arousal with intermediate valence), and half were negative (high arousal with low valence). ANEW stimuli are rated on a 1-8 scale for valence (increasingly more positive) and a 1-8 scale for arousal (increasingly more arousing). All the neutral words used here had valence ratings between 4.5 and 6 , with arousal ratings between 2.5 and 4 . All the negative words used had valence ratings below 3 , with arousal ratings above 6 . These choices for neutral and highly arousing negative words were very similar to those in Marx et al. (2008), who found a lack of suppression for highly arousing negative words. All the word stimuli were in 64-point Arial font presented in the center of the screen. Word length, word frequency, and semantic relatedness were controlled between and among pairs. All of the individual words were chosen so as to not be readily meaningfully associated, and the specific pairs themselves were further chosen so as to not represent common associations. The experimenters originally chose the words with feedback on relatedness from an independent judge. Furthermore, word pairs were counterbalanced between the suppression and recall conditions between participants.

\section{General Procedure}

The entire experiment lasted $2-2.5 \mathrm{~h}$ and consisted of three main phases. Following informed consent, the first phase involved the participants' encoding cue-target pairs of words outside the imaging environment. The second phase involved functional imaging of the participants while they were shown cue words and were signaled that they had to either recall or suppress target items. Lastly, the third phase involved memory tests and questionnaires given outside the imaging environment.

Phase 1: Word pair encoding. The prescan training involved the participants' learning all of the word pairs such that they could respond to the cue words, in the necessary time allotted, with the correct targets. Word pairs were randomly presented on a computer screen. First, the participants were exposed to all of the word pairs, referred to as cues and targets. Next, they were shown the cues paired with a question mark and were told to respond as quickly and accurately as possible with the appropriate target word. After $2 \mathrm{sec}$, the target word was presented next to the cue word. The experimenter sat next to the participants and measured the accuracy across all repetitions. This continued until all the targets could be recalled accurately within the allotted time of $2 \mathrm{sec}$. The participants were given at least 6 repetitions of all the words, and no participant required more than 10 repetitions (except for the individual excluded from the analyses). The fact that word pair learning was brought to ceiling should help eliminate discrepancies in memory performance due to differences in the general semantic relatedness of neutral versus negative targets. One potential issue with this method is that there may have been differences in the speed of learning for neutral versus negative pairs. We tracked the learning of all of the pairs for every participant and, thus, were able to measure the speed of learning for neutral versus negative pairs.

Phase 2: Critical recall/suppression imaging phase. After the participants had learned all of the pairs, they were instructed on the next phase of the experiment. This phase was equivalent to the critical TNT phase. The participants were told that they would see the cue words in either a blue or a yellow font. This color would signify that they should either recall the target or avoid recalling the target. The font color instruction and the specific items recalled or suppressed were counterbalanced among participants. Again, all cues were neutral, in order to control for the perception of emotion during this phase. 
For the suppress cues, the participants were specifically told to "avoid the recall of the target" and to "keep the target out of awareness or consciousness." To help control for the strategy used during the suppression, a specific strategy was suggested. If they were in a no-think trial, the participants were told to attempt to read the cue word backward, to help them not think of the target word. The participants were also told that if this strategy was unsuccessful, they could try another, similar strategy that required them to focus on the physical aspects of the cue word and distract their attention away from recalling the target.

Functional imaging consisted of six runs lasting approximately 4 min each (see Figure 1 for a schematic of the design). The participants saw all 40 cues during each run; half the cues were presented in yellow font, and half in blue. The order of the cues corresponding to the specific conditions was randomized. The cues were presented for $2 \mathrm{sec}$ each and were followed by a variable interstimulus interval (ISI) of 2, 4, or $6 \mathrm{sec}$ (see Figure 1). After all six functional runs had been completed, a high-resolution anatomical scan was administered.

Imaging parameters. Imaging was performed using a 3-T Siemens Magnetom Trio whole-body MRI system and a phased array eight-channel head coil, located at the Indiana University Psychological and Brain Sciences department. All the stimuli were backdisplayed via a Mitsubishi XL30 projector onto a screen that was viewed through a mirror from the bore of the scanner. The stimuli were presented via SuperLab Pro 2.0.4 software via a Macintosh Macbook laptop.

The field of view was $22 \times 22 \times 9.9 \mathrm{~cm}$, with an in-plane resolution of $64 \times 64$ pixels and 33 slices per volume that were $3.4 \mathrm{~mm}$ thick. These parameters allowed us to collect data from the entire brain. The resulting voxel size was $3.4 \times 3.4 \times 4.0 \mathrm{~mm}$. Images were acquired using an echo-planar technique $(\mathrm{TE}=30 \mathrm{msec}$, $\mathrm{TR}=2,000 \mathrm{msec}$, flip angle $=90^{\circ}$ ) for BOLD-based imaging. High-resolution T1-weighted anatomical volumes were acquired using a 3D Turbo-flash acquisition. Functional data underwent slice time correction, 3-D motion correction, linear trend removal, and Gaussian spatial blurring (FWHM $6 \mathrm{~mm}$ ), using the analysis tools in Brain Voyager. Individual functional volumes were coregistered to anatomical volumes with an intensity-matching, rigid-body transformation algorithm. Individual anatomical volumes were normalized to the stereotactic space of Talairach and Tournoux (1988), using an eight-parameter affine transformation, with parameters selected by visual inspection of anatomical landmarks. Applying the same affine transformation to the coregistered functional volumes placed the functional data in a common brain space, allowing comparisons across participants. Voxel size of the normalized functional volumes was standardized at $1 \times 1 \times 1 \mathrm{~mm}$, using trilinear interpolation.
Phase 3: Postscan memory test and questionnaire. The postscan testing included cued recall tests of all the word pairs. The cued recall was performed to check that the participants remembered the pairs and, thus, increase confidence that the word pairs were successfully recalled during the critical phase. A performance and strategy questionnaire based on Hertel and Calcaterra (2005) was given as well. It measured both compliance with strategy and self-reports of performance.

\section{Performance and Strategy Controls}

Performance control. We were predominantly interested in investigating the neural networks differentially activated during the attempted suppression of negative, as compared with neutral, items. Previous TNT fMRI studies have used subsequent memory to separate failed versus successful recall and suppression. In order to avoid subsequent ceiling effects, participants have been trained to recall only a percentage of the targets when shown the cues. However, we chose to ensure that the participants could recall all of the targets before beginning the critical suppress/recall phase. Thus, in the training phase, the participants learned all of the pairs, so that they were able to recall the target when shown the cue within the same time $(2 \mathrm{sec})$ as that allotted during the critical suppress/recall phase. They were subsequently tested to ensure that they were able to recall the targets correctly while they were in the scanner. Although separating success and failure in these studies is important, some results have been found to be very similar whether or not subsequent performance was taken into account (Depue et al., 2007, and the supplemental materials). The presence or absence of a subsequent behavioral effect does not preclude one from focusing on the active processes involved in attempts to avoid recall of the targets, using functional imaging methods. This was the focus of the present study.

Strategy control. Usually during suppression in the TNT paradigm, the participants were allowed to use whatever strategy they wished, potentially adding variability to cognitive processes during this phase (Anderson et al., 2004; Depue et al., 2007). The strength of this approach is that it allows for more general conclusions in regard to suppression. However, a problem arises when multiple types of targets are used at once, as in the present study. More specific conclusions are drawn from the imaging results across target types when they are based on a more defined strategy. In one sense, this is a limitation, because it reduces generalizability. It was deemed crucial for this initial study in which the suppression of neutral stimuli was compared directly with that of negative stimuli, because differences in strategy between neutral and negative targets would confound the functional imaging results. It would be unclear whether differences in activation were due to differences in the nature of the targets, differences in the strategy used to suppress neutral versus negative targets, or both of these possibilities. For example, indi-

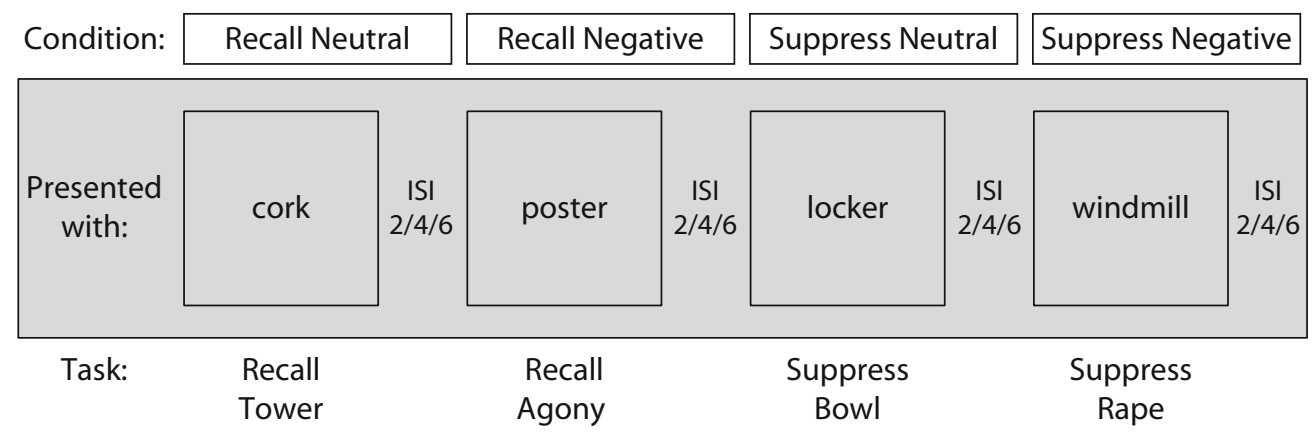

Figure 1.fMRI procedure. After learning all the pairs (examples: cork-tower, poster-agony,locker-bowl, windmill-rape), the participants were moved to a scanner and shown cues for 2 sec each. Interstimulus intervals (ISIs) were varied at 2,4 , and 6 sec. The cues were in either a blue or a yellow font (counterbalanced), indicating an instruction to either recall or suppress the associated target word. All the targets were either recalled or suppressed six times total across six separate runs. 
viduals may use emotion regulation strategies for negative, but not neutral, suppression targets. We would not be able to pull apart these strategy differences from differences due purely to the emotionality of the cue. However, in the present study, this limits generalizing to suppression in general. The results are limited to a strategy in the realm of attentional distraction. Because of the instructed strategy, it should be made clear that the results are specific to suppression attempted through orthographic analysis.

\section{fMRI Data Analysis Procedures}

The data were deconvolved without using an assumed hemodynamic response function. Statistical parametric maps (SPMs) were created using the random-effects multistudy general linear model procedure. Four main whole-brain contrasts of interest were performed that compared the effects of emotion (neutral or negative) and instruction (recall or suppress). The first two contrasts compared activation of suppression versus recall isolated within a specific emotional level. These included contrasts of suppression of neutral $>$ recall of neutral and suppression of negative $>$ recall of negative. The third contrast, recall of neutral $>$ recall of negative, was created to investigate whether differences between the first two contrasts might be due to differences in the recall, and not the suppression, conditions. The fourth contrast, suppression of negative $>$ suppression of neutral, was created to directly test the differences between the suppression of neutral and negative items. All group contrasts were evaluated at a statistical threshold of $p<.001$ (uncorrected).

Further analyses involved investigating individual responses in specific regions of interest (ROIs). Anatomical ROIs were defined in the whole left and right hippocampus, the posterior left and right hippocampus, the anterior left and right hippocampus, and the whole left and right amygdala. Using the segmentation tool provided in Brain Voyager, the given area was drawn in each individual participant's untransformed brain (nonnormalized) to allow for more accurate representations of individual anatomical/structural differences. This segmentation was entirely manual. Pruessner et al. (2000) was referenced for standardized methods of manual segmentation. This work describes methods for segmenting the hippocampal tail, body, and head, as well as the amygdala. For the purposes of this study, the posterior hippocampus included the hippocampal tail, and the anterior hippocampus included the hippocampal body and head. Also, an anatomical reference guide (Duvernoy, Bourgouin, \& Vannson, 1999) was consistently referenced during this process. The functional data overlaid on the nonnormalized anatomical data were preprocessed in the same way as the data used for the wholebrain analysis.

Data from all six runs combined, as well as Runs 1, 2, and 3 isolated (early runs) and Runs 4, 5, and 6 isolated (late runs), were extracted from these ROIs. Within-subjects repeated measures ANOVAs, comparing instruction (recall or suppress) and emotion (neutral or negative), were performed using the peak BOLD response of the time course of each ROI as the dependent measure. The peak BOLD analysis was chosen to equalize individual variability in the timing of neural and cognitive processes. Neural activation can peak at different times among individuals; in one sense, this is informative if one is interested in timing differences among individuals. Here, however, we were more interested in the comparison of energy requirements of brain regions during our conditions; these energy requirements were assumed to be reflected by peak BOLD activation. However, a range within which peak activation was considered to reflect energy requirements was set to be 3-12 sec post-stimulus-onset. This dependant measure was acquired from a time course of data averaged over all voxels in a given ROI, and not a single peak voxel in the ROI. Two alternate ROI analyses were performed and yielded results similar to those of the peak BOLD response analysis. The first was an analysis in which, instead of the peak from 3-12 sec poststimulus, that entire time range was averaged for all the participants. This analysis is important because it is more comparable to what has been done in previous fMRI TNT studies (Anderson et al., 2004; Depue et al., 2007). The second was an analysis using the average of $6 \mathrm{sec}$ of data around the peak in the same range of 3-12 sec poststimulus. Averaging of the entire time course and of the $6 \mathrm{sec}$ around the peak did not change the results significantly, supporting the usefulness of peak selection analyses. These additional analyses can be found in the supplemental materials.

\section{RESULTS AND DISCUSSION}

\section{fMRI SPM Contrasts}

fMRI results suggested that the hippocampus (bilaterally) was relatively less active during the suppression of neutral, but not negative, words. This is the first demonstration of such a difference between the suppression of neutral and negative memories. The results of contrasting BOLD activation during suppression of neutral words with activation during recall of neutral words are presented in Figure 2A. Table 1 displays the results in terms of significantly activated clusters from this and all the other contrasts discussed.

Analyses showed greater frontoparietal activation during suppression than during recall, as well as less activation in the posterior hippocampus during suppression of neutral words, as compared with the recall of neutral words (Figure 2A, Slices I-V). This pattern of results is consistent with that found by Anderson et al. (2004). Crucially, in the contrast of suppression versus recall of negative words, the decrease in hippocampal activation during suppression was no longer demonstrated (Figure 2B, Slices IV and V). Common to both contrasts was the greater activation in the frontoparietal network during suppression (Figures 2A and 2B, Slices I, II, and III). Very similar brain regions were seen in both of these orthogonal contrasts. This might be expected if the same strategy was employed for both neutral and negative targets. Interestingly, this activity was right lateralized, meshing with evidence that righthemispheric frontal lesions lead to deficits in intentional forgetting (Conway \& Fthenaki, 2003). Depue et al. (2007) also indicated right-lateralized frontal regions in the control of emotional memories. Go/no-go studies have revealed similar right-lateralized patterns related to motor inhibition as well (Garavan, Ross, \& Stein, 1999). The findings of the present study further suggest that frontoparietal areas may be involved in the strategies used to attempt suppression of recall, and possibly the modulation of the hippocampus (Anderson et al., 2004; Depue et al., 2007).

Increased hippocampal activation at encoding (Fernández et al., 1999) and retrieval (Cabeza, Rao, Wagner, Mayer, \& Schacter, 2001; Eldridge, Knowlton, Furmanski, Bookheimer, \& Engel, 2000) has been associated with successful conscious recall. Previous studies using the TNT paradigm with fMRI have shown decreased hippocampal activation to be associated with suppression of recall (Anderson et al., 2004; Depue et al., 2007). The results above indicate that relatively lower hippocampal activation did not occur during the suppression of negative items. In light of the previous studies, this might reflect the greater recollection of negative words in spite of attempts to suppress 


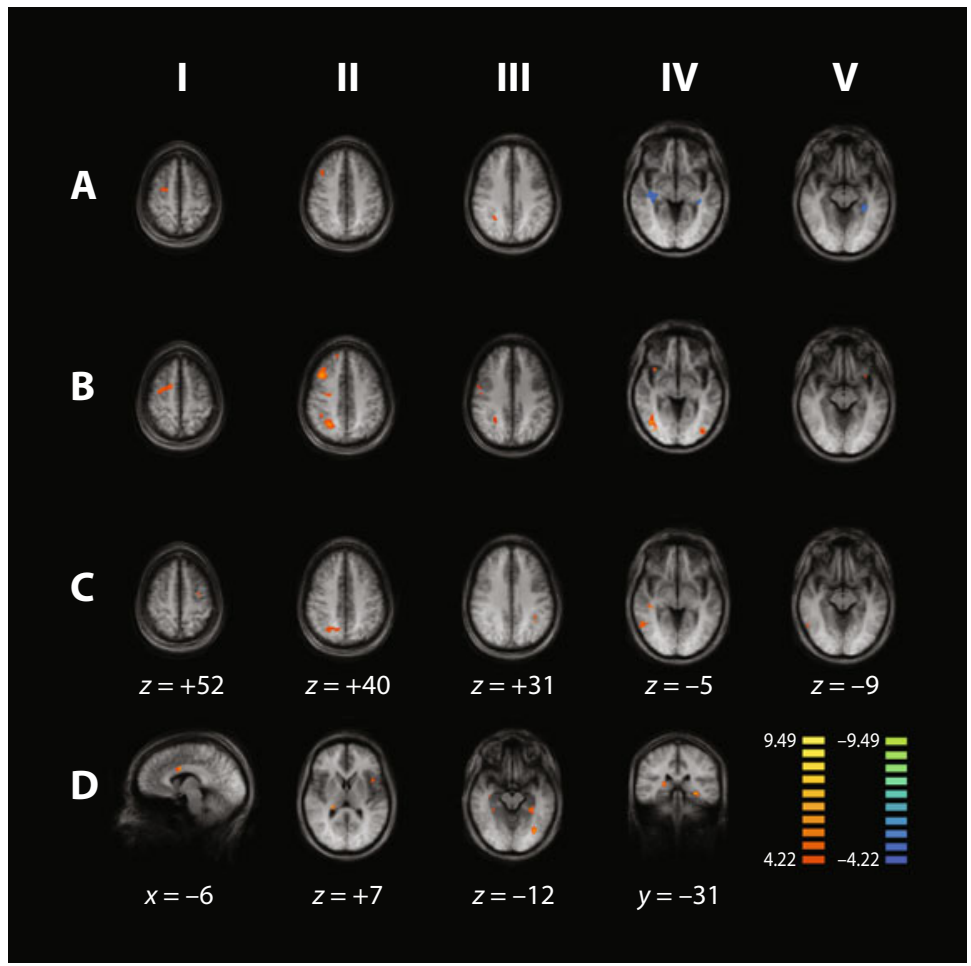

Figure 2. Whole-brain direct contrasts, showing each contrast of interest for reference and comparison. The contrasts are presented at a threshold of $p<.001$, uncorrected. The bottom right shows the color coding of the $t(13)$ values. Images are shown in radiological conventions (right is left). Relevant Talairach coordinates are shown below the images. (A) Contrast of suppress neutral $>$ recall neutral. (B) Contrast of suppress negative $>$ recall negative. (C) Contrast of recall neutral $>$ recall negative. (D) Contrast of suppress negative $>$ suppress neutral (see the text for a further description).

recall. It may also represent increased intrusions of the target word during suppression. The third contrast of interest compared the two recall conditions in order to further test whether the differences of note observed in the previous two contrasts could be attributed to differences across emotion during recall alone. In this recall contrast (recall neutral $>$ recall negative), several areas were relatively more active during recall neutral than during recall negative (Figure $2 \mathrm{C}$, Slices I-V). Importantly, none of these differences were found in the medial temporal lobes. This suggests that in this region, differences observed between suppression and recall conditions in the first two contrasts (Figures 2A and $2 \mathrm{~B}$, Slices IV and V) may be attributed to differences in the level of emotion during suppression attempts.

Further confirmation of the results above was found in the direct contrast of suppression of negative words versus neutral words (Figure 2D, Slices III and IV), revealing greater hippocampal activation during the suppression of negative words. Several other areas were relatively more activated during attempts to suppress negative items than during attempts to suppress neutral items. The anterior cingulate cortex (ACC) was more active during the suppression of negative words than during the suppression of neutral words (Figure 2D, Slice I). The ACC has been found to be involved in conflict and error detection and in signaling the need for greater cognitive control (Botvinick, Cohen, \& Carter, 2004; Lutcke \& Frahm, 2008). Greater activation of the ACC during the suppression of negative words may point to a greater need for control when negative words are suppressed, because of greater conflict between the automatic recall and controlled suppression processes. Another possibility is that there were more overall failures during attempts at suppressing negative words, leading to greater errors being detected. Brown and Braver (2005) suggested that, overall, the ACC activates more generally in cases in which the likelihood of error is predicted to be greater. Thus, suppression of errors, in the form of intrusions of recall, may be more likely for negative targets.

The left insula showed greater activation during the suppression of negative items than during the suppression of neutral items (Figure 2D, Slice II). Past imaging studies have shown insular activation during both cognitively demanding emotional tasks and recall of emotional material (Phan, Wager, Taylor, \& Liberzon, 2002). Others have shown that the insula plays a role in evaluating emotional responses and, specifically, distressful thoughts (Reiman et al., 1997). Stop signal paradigms have found greater activation in the insula during failed attempts to inhibit motor responses (Ramautar, Slagterb, Koka, \& Ridderinkhof, 2006). The involvement of the insula here 
Table 1

Whole-Brain Direct Contrast Results, Showing the Locations of All Significant Clusters From the Contrasts of Interest

\begin{tabular}{|c|c|c|c|c|c|}
\hline Contrast & Cluster Location & $\begin{array}{l}\text { Talairach Coordinates } \\
\text { (Peak) }(x, y, z)\end{array}$ & $\begin{array}{l}\text { Cluster Size } \\
\text { (1-mm Voxels) }\end{array}$ & $t(13)$ Peak & $p<$ (Uncorrected $)$ \\
\hline NTneut $>$ Tneut & $\begin{array}{l}\text { Right middle frontal gyrus BA } 46 \\
\text { Right inferior parietal lobule BA } 39 / 40 \\
\text { Right postcentral gyrus BA } 6 \\
\text { Left precentral gyrus BA } 4\end{array}$ & $\begin{array}{l}36,20,40 \\
27,-49,31 \\
27,-7,55 \\
-51,-4,19\end{array}$ & $\begin{array}{r}58 \\
159 \\
282 \\
134\end{array}$ & $\begin{array}{l}4.40 \\
6.17 \\
4.86 \\
4.86\end{array}$ & $\begin{array}{l}.000715 \\
.000034 \\
.000313 \\
.000310\end{array}$ \\
\hline Tneut $>$ NTneut & $\begin{array}{l}\text { Right posterior hippocampus BA N/A } \\
\text { Right precuneus BA } 7 \\
\text { Left posterior hippocampus } 6 \text { BA N/A }\end{array}$ & $\begin{array}{l}36,-31,-2 \\
15,-55,16 \\
-30,-34,-11\end{array}$ & $\begin{array}{r}422 \\
79 \\
462\end{array}$ & $\begin{array}{l}-5.90 \\
-5.53 \\
-7.80\end{array}$ & $\begin{array}{l}.000053 \\
.000097 \\
.000003\end{array}$ \\
\hline NTneg $>$ Tneg & $\begin{array}{l}\text { Right superior temporal gyrus BA } 22 \\
\text { Right inferior frontal gyrus BA } 47 \\
\text { Right precentral gyrus BA } 4 \\
\text { Right superior temporal gyrus BA } 22 \\
\text { Right middle occipital gyrus BA } 18 \\
\text { Right middle frontal gyrus BA } 46 \\
\text { Right inferior parietal lobule BA } 39 / 40 \\
\text { Right postcentral gyrus BA } 6 \\
\text { Right superior frontal gyrus BA } 9 \\
\text { Left middle occipital gyrus BA } 18 \\
\text { Left fusiform gyrus BA } 37 \\
\text { Left middle temporal gyrus BA } 21 \\
\text { Left precentral gyrus BA } 6\end{array}$ & $\begin{array}{l}51,-25,1 \\
54,20,16 \\
42,-10,28 \\
48,-55,-17 \\
36,-73,1 \\
39,17,40 \\
24,-52,37 \\
36,-7,46 \\
21,26,31 \\
-33,-76,1 \\
-36,-55,-14 \\
-48,-40,4 \\
-54,-1,13\end{array}$ & $\begin{array}{r}467 \\
1,265 \\
542 \\
329 \\
1,311 \\
1,873 \\
2,448 \\
1,783 \\
453 \\
291 \\
964 \\
277 \\
564\end{array}$ & $\begin{array}{l}8.97 \\
7.17 \\
6.04 \\
4.80 \\
6.09 \\
7.42 \\
6.68 \\
6.11 \\
5.39 \\
5.60 \\
5.92 \\
8.49 \\
6.27\end{array}$ & $\begin{array}{l}.000001 \\
.000007 \\
.000042 \\
.000349 \\
.000039 \\
.000005 \\
.000015 \\
.000037 \\
.000124 \\
.000086 \\
.000051 \\
.000001 \\
.000029\end{array}$ \\
\hline Tneut $>$ Tneg & $\begin{array}{l}\text { Right middle temporal gyrus BA } 21 \\
\text { Right caudate BA N/A } \\
\text { Right middle temporal gyrus } 21 \\
\text { Right inferior frontal gyrus BA } 44 \\
\text { Right middle occipital gyrus BA } 18 \\
\text { Right insula BA 13 } \\
\text { Right precuneus BA } 7 \\
\text { Left precuneus BA } 7 \\
\text { Left precentral gyrus BA } 4 \\
\text { Left inferior parietal lobule BA } 39 / 40\end{array}$ & $\begin{array}{l}51,-55,-5 \\
39,-28,-5 \\
48,-64,7 \\
42,23,16 \\
30,-85,13 \\
30,23,10 \\
9,-61,46 \\
-15,-64,37 \\
-30,-10,52 \\
-33,-40,25\end{array}$ & $\begin{array}{l}328 \\
239 \\
198 \\
180 \\
274 \\
163 \\
633 \\
176 \\
159 \\
351\end{array}$ & $\begin{array}{l}5.05 \\
6.56 \\
5.27 \\
6.43 \\
5.49 \\
6.27 \\
5.38 \\
5.41 \\
5.15 \\
6.41\end{array}$ & $\begin{array}{l}.000222 \\
.000018 \\
.000152 \\
.000022 \\
.000104 \\
.000029 \\
.000125 \\
.000120 \\
.000187 \\
.000023\end{array}$ \\
\hline NTneg $>$ NTneut & $\begin{array}{l}\text { Right inferior frontal gyrus BA } 44 \\
\text { Right fusiform gyrus BA } 37 \\
\text { Right parahippocampal gyrus BA } 36 \\
\text { Right pulvinar of the thalamus BA N/A } \\
\text { Left anterior cingulate gyrus BA 23 } \\
\text { Left posterior hippocampus BA N/A } \\
\text { Left fusiform gyrus BA } 37 \\
\text { Left anterior insula BA } 13\end{array}$ & $\begin{array}{l}54,5,25 \\
39,-46,-23 \\
30,-34,-11 \\
21,-31,7 \\
-9,5,31 \\
-27,-31,-8 \\
-33,-67,-11 \\
-42,11,7\end{array}$ & $\begin{array}{r}116 \\
190 \\
77 \\
240 \\
198 \\
905 \\
876 \\
56\end{array}$ & $\begin{array}{l}4.95 \\
6.70 \\
4.90 \\
7.08 \\
6.64 \\
7.68 \\
6.67 \\
4.55\end{array}$ & $\begin{array}{l}.000268 \\
.000015 \\
.000291 \\
.000008 \\
.000016 \\
.000003 \\
.000015 \\
.000546\end{array}$ \\
\hline
\end{tabular}

may correlate with increased failed suppression of negative words that are associated with increased negative affect or feelings.

During the suppression of negative versus neutral words, greater activation was also discovered in the left fusiform gyrus (Figure 2D, Slice III). Interestingly, the area in the left fusiform gyrus that showed greater activation during the suppression of negative words than during the suppression of neutral words was near the visual word form area (Fiez \& Petersen, 1998). This area is thought to be involved in the visual perception of words (McCandliss, Cohen, \& Dehaene, 2003). The fact that words were presented on the screen in both conditions used in this contrast suggests that this effect may be related to memory

Table 2

Speed-of-Learning ANOVA Results

\begin{tabular}{lcc}
\hline \multicolumn{1}{c}{ Result } & $F(1,13)$ & $p$ \\
\hline Main effect of task (recall/suppression) & 0.903 & .359 \\
Main effect of emotion (neutral/negative) & 1.621 & .225 \\
Interaction of task and emotion & 0.808 & .385 \\
\hline
\end{tabular}

processes. Previous studies have reported reactivation of sensory areas during memory recall (Slotnick, 2004). Furthermore, this reactivation has been shown to be modality specific, such that recall of words paired with auditory stimuli or visual stimuli shows reactivation in secondary auditory and visual regions, respectively (Khader, Burke, Bien, Ranganath, \& Rösler, 2005; Wheeler, Petersen, \& Buckner, 2000). This is thought to occur because perceptual areas, involved in encoding, become important aspects of the memory trace or representation necessary for recollection (Vaidya, Zhao, Desmond, \& Gabrieli, 2002). The fact that this activity was greater during the suppression of negative targets than during the suppression of neutral targets suggests relatively more intrusive recollections during attempts to suppress emotional target information.

Several of the areas that were found to be more greatly activated during the suppression of negative, as compared with neutral, targets are similar to the areas downregulated in association with successful suppression of emotional target memories in Depue et al. (2007). These include the hippocampus, thalamus, and visual regions 
Whole Left Hippocampus

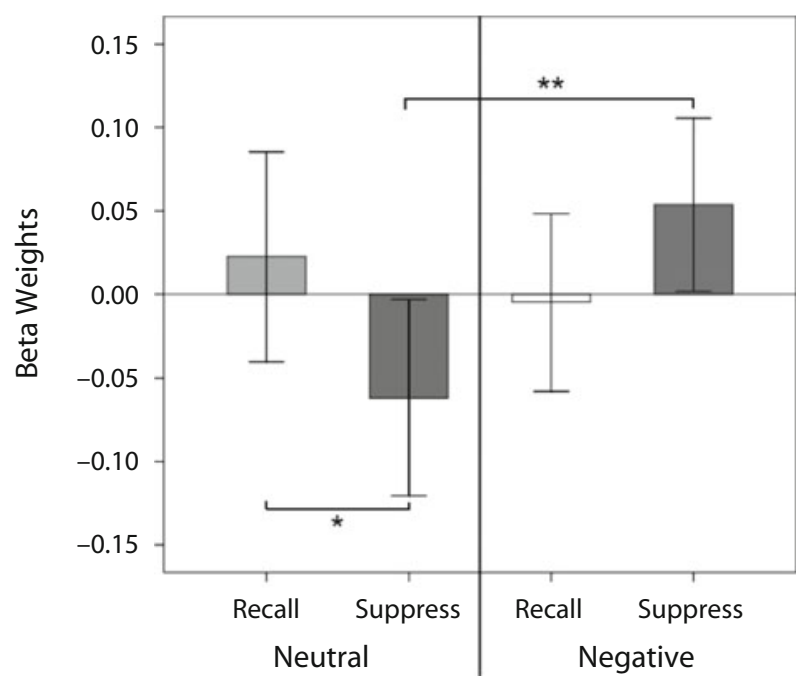

Posterior Left Hippocampus

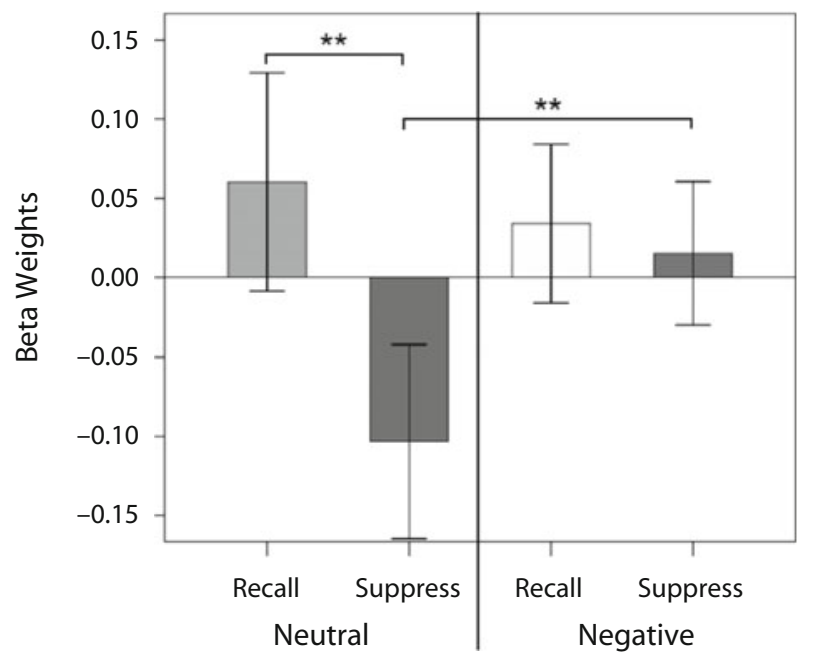

Whole Right Hippocampus

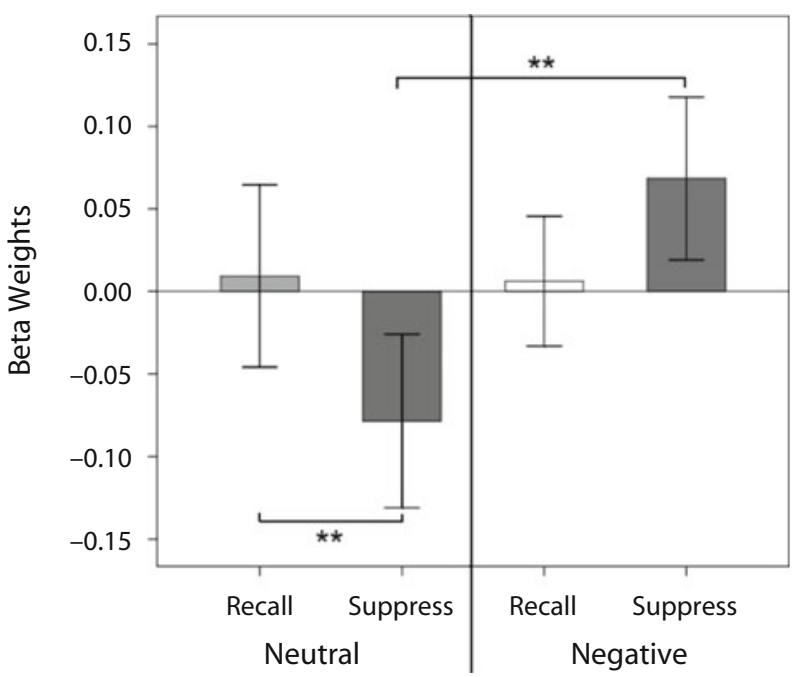

Posterior Right Hippocampus

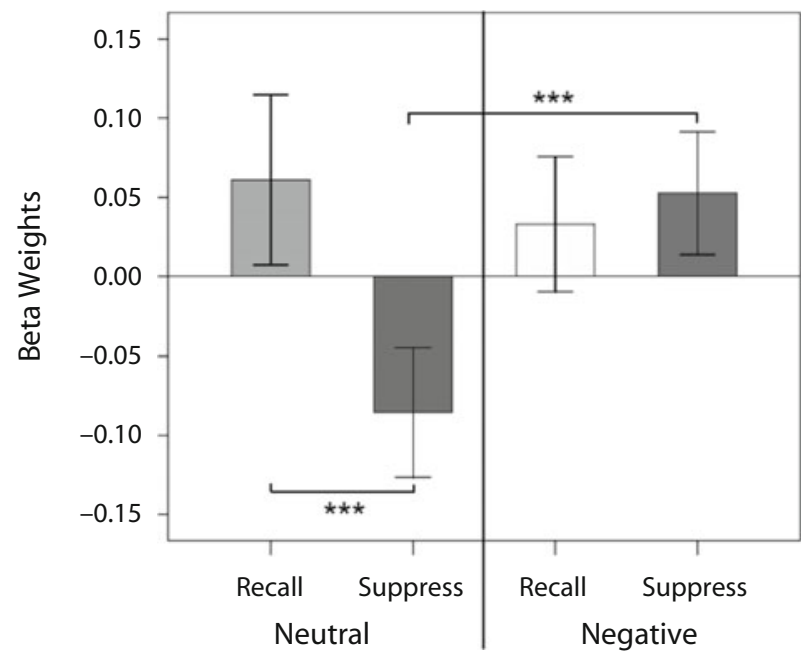

Figure 3. Anatomical region-of-interest results in both the whole and posterior bilateral hippocampus from all run repetitions combined. The whole and posterior bilateral hippocampus shows lowered activation for the suppression of neutral words, as compared with the recall of neutral words. However, no differences were found between the recall and suppression of negative words. Beta weights represent percent BOLD signal change. Bars represent mean peak BOLD response for each condition. Error bars represent 1 standard error of the mean. Significance bars represent ${ }^{*} p<.05,{ }^{* *} p<.01$, and ${ }^{* * *} p<.001$.

(Figure 2D, Slices II, III, and IV). This further suggests that these regions are important for the representation of emotional memories.

\section{ROI Analysis and}

\section{Effect of Repeating Suppression}

The anatomical ROI analysis supported and further extended the findings of the SPM analyses. The results, combining all six runs, revealed differences between emotion (neutral vs. negative) and instruction (recall vs. suppression). This analysis allowed for greater anatomical specificity. It also compensated for the large amount of multiple comparisons present in whole-brain voxelwise analyses. Figure 3 shows the results from the whole and posterior bilateral hippocampus. A repeated measures ANOVA revealed a significant interaction in the whole left hippocampus $[F(1,13)=7.855, p<.05]$, as well as in the whole right hippocampus $[F(1,13)=7.392, p<$ $.05]$. Post hoc $t$ tests revealed significant differences between recall neutral and suppress neutral conditions for the whole left hippocampus $[t(13)=2.161, p<.05]$ and for the whole right hippocampus $[t(13)=3.013, p<.01]$, such that suppression led to a decreased mean response for neutral words. In addition, post hoc $t$ tests revealed significant differences between suppress neutral and suppress negative conditions for the whole left hippocampus 
$[t(13)=3.013, p<.01]$ and for the whole right hippocampus $[t(13)=3.013, p<.01]$, such that suppression of negative words showed greater response than did the suppression of neutral words.

A repeated measures ANOVA revealed that a significant interaction was found in the posterior left hippocampus $[F(1,13)=4.538, p<.05]$ and the posterior right hippocampus $[F(1,13)=7.781, p<.05]$. Post hoc $t$ tests revealed significant differences between recall neutral and suppress neutral conditions $[t(13)=3.013, p<.01$, for the posterior left hippocampus, and $t(13)=4.220$, $p<.001$, for the posterior right hippocampus], such that suppression led to decreased mean response for neutral words. In addition, post hoc $t$ tests revealed significant differences between suppress neutral and suppress negative conditions $[t(13)=3.013, p<.01$, for the posterior left hippocampus, and $t(13)=4.220, p<.001$, for the posterior right hippocampus], such that suppression of negative words showed greater response than did the suppression of neutral words.

The anterior bilateral hippocampus did not show this same interaction. Although a repeated measures ANOVA revealed that the interaction of emotion and instruction approached significance in the left anterior hippocampus $[F(1,13)=3.654, p=.078]$ and the right anterior hippocampus $[F(1,13)=3.977, p=.068]$, the interaction was due to greater activation during the suppression of negative words, as compared with recall of negative words, with no differences between recall and suppress neutral. The analysis further revealed that both the left amygdala $[F(1,13)=2.974, p=.108]$ and the right amygdala $[F(1,13)=0.497, p=.493]$ showed no significant differences across conditions when all six runs/repetitions were considered.

The comparison of early repetitions (including Runs 1,2, and 3) with later repetitions (including Runs 4, 5, and 6) allows us to see the effect of multiple repetitions of suppression and recall on responses in these areas. Figure 4 shows the results of this analysis in the whole left hippocampus and left amygdala. Increasing repetitions of suppression have been associated with greater forgetting (Anderson \& Green, 2001) and decreased hippocampal activation (Depue et al., 2007). An interaction between emotion and instruction (recall vs. suppression) was found in the whole left hippocampus during late $[F(1,12)=7.01, p=.021]$, but not early $[F(1,13)=2.011, p=.18]$, runs in which suppress neutral was significantly decreased from recall neutral [post hoc $t(12)=2.179, p<.05$ ], but no differences were found between suppress and recall negative. This same general pattern was found in the whole right hippocampus, as well as the posterior left and right hippocampus. These results support the role of increasing repetitions of suppression leading to greater hippocampal deactivation. However, increased repetitions of suppression lead to a greater decrease in hippocampal activation for neutral, but not negative, words.

Although no differences were found in the amygdala when the data from all six repetitions (i.e., across all six runs) were combined, there was a significant interaction between emotion and instruction in the left amygdala dur- ing later repetitions $[F(1,12)=4.614, p<.05]$. A post hoc $t$ test revealed greater activation during the suppression of negative words, as compared with the recall of negative words $[t(12)=2.179, p<.05]$, but no significant differences between the suppression and recall of neutral words. The amygdala has generally been implicated in emotional processing (Phelps \& LeDoux, 2005). The amygdala is important in the modulation of memory by emotion (Greenberg et al., 2005; LaBar \& Cabeza, 2006; Phelps, 2004). Activation in the amygdala coincides with the reexperience of emotion during retrieval (Buchanan, 2007), especially for personally experienced traumatic events (Sharot, Martorella, Delgado, \& Phelps, 2007). fMRI has revealed that memory recall is enhanced for emotional items (Kensinger \& Schacter, 2005), especially when the amygdala and hippocampus show coactivation (Dolcos, LaBar, \& Cabeza, 2004).

Figure 4 shows the results from the whole left hippocampus and left amygdala, comparing early with late repetitions. As in Depue et al. (2007), the hippocampus showed a significant decrease in activation over time. However, during later repetitions, the suppression of negative words was not associated with a decrease in hippocampal response, as were the neutral words. Furthermore, the suppression of negative words was associated with increased left amygdalar activation during this same later time period. It is possible that amygdalar activation may contribute to differences in hippocampal modulation during the suppression of negative words, as compared with neutral words. Attempts to suppress negative memories may require the modulation of both the hippocampus and amygdala (Depue et al., 2007).

\section{Performance and Strategy}

Overall, the participants were near ceiling for postscan cued recall (96\% correct), as was expected. Performance was thus equated across levels of emotion (neutral/ negative) and instruction (recall/suppression) both before and after the critical recall suppression phase in the imaging environment, suggesting that the participants were able to recall the target words that were presented during the critical phase that occurred in the scanner.

The specific strategy used during suppression is difficult to study, but it is of the utmost importance. Thought substitution has been found to be a successful strategy in that it increased the level of intentional forgetting in the TNT paradigm (Hertel \& Calcaterra, 2005). None of the TNT fMRI studies to date have controlled or reported suppression strategy. There is a seemingly large amount of strategies that could be used. It is understandable that one would not want to hinder performance with a forced strategy. On the other hand, it is likely that different strategies could lead to differential effects on behavior and would very likely be reflected in strategy-dependent brain activation. Crucially, differences in the suppression strategy employed between neutral and negative targets could have large effects on strategy-related activation. These differences would be hard to distinguish from activation specifically associated with the emotionality of the target. In the present study, we provided a suggested strategy in 


\section{Whole Left Hippocampus \\ Early Repetitions}

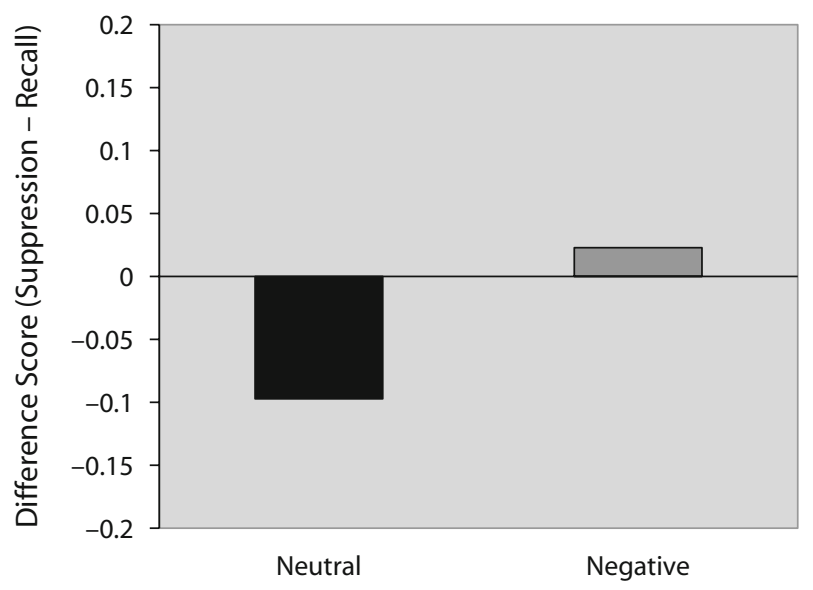

Emotion

Left Amygdala

Early Repetitions

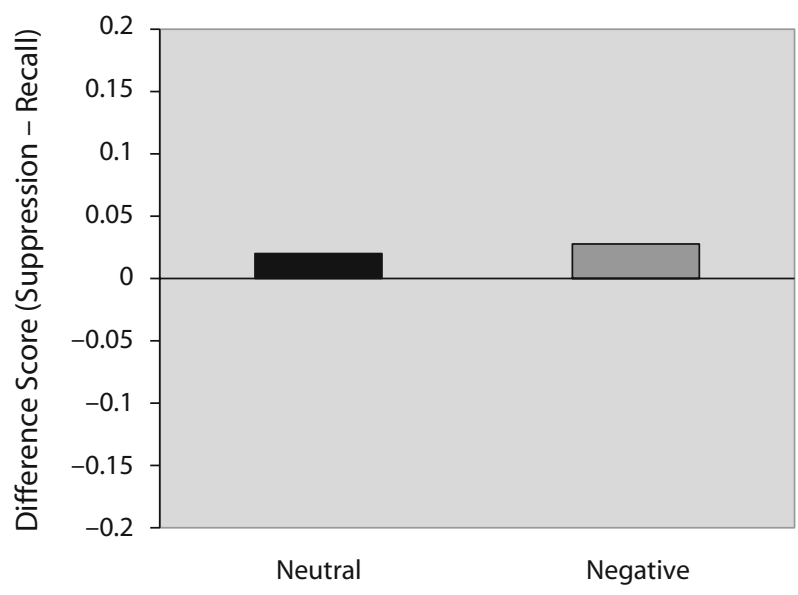

Emotion
Whole Left Hippocampus Late Repetitions

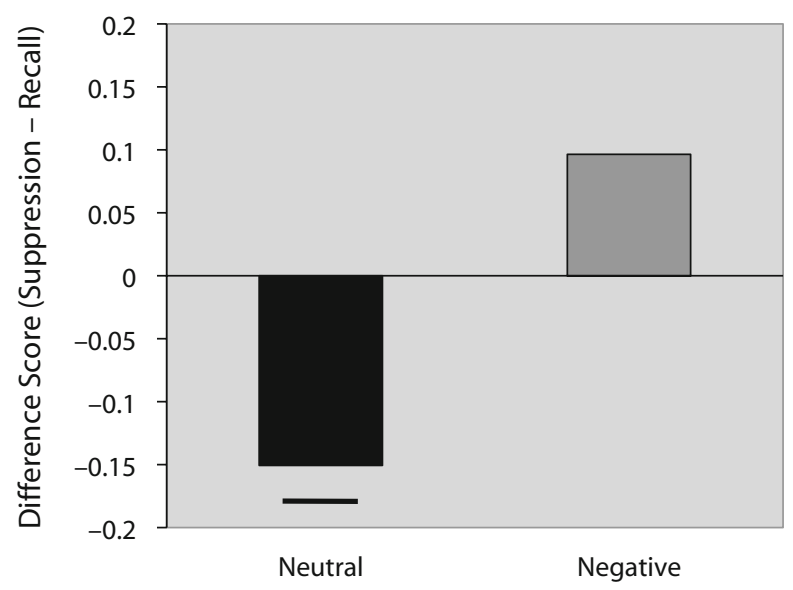

Emotion

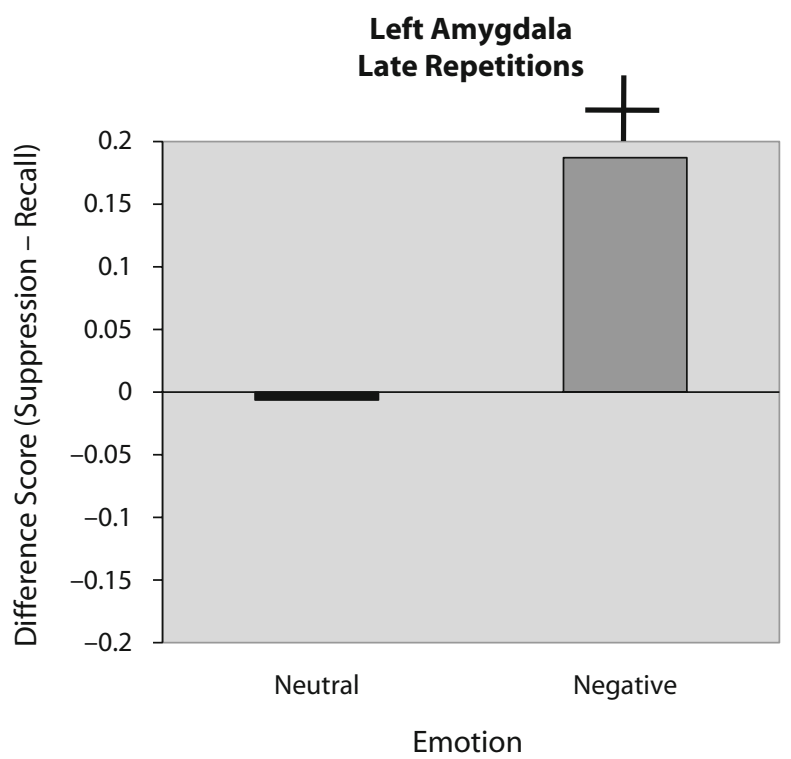

Figure 4. Comparison of early (Runs/Repetitions 1, 2, and 3 combined) with late (Runs/Repetitions 4, 5, and 6 combined) suppression repetitions in the whole left hippocampus and left amygdala. The bars represent a difference score of the mean peak BOLD response for suppression subtracted from the mean response for recall within a given level of emotion. This indexes the amount of decrease or increase in the BOLD response for suppression, relative to recall. The - and + symbols represent a significant difference between suppression and recall in the negative and positive directions, respectively. The left hippocampus showed a significant decrease for suppression of neutral words during late, but not early, runs, with no difference for negative words. The amygdala showed a significant increase during the suppression of negative words, but not neutral words, in later repetitions.

the attempt to control potential variability across participants and across target types. Again, this may help control across the neutral and negative conditions at the expense of limiting generalization. The participants were tested after the experiment and reported compliance with this general strategy. The performance and strategy questionnaire revealed that 9 of the participants reported using the suggested backward cue-reading strategy. Other participants reported using strategies that involved using the cue word in order to distract from recalling the target word (i.e., unscrambling the letters in the cues or repeating let- ters in the cues). No participants reported using different strategies for the cue with neutral versus negative targets. This supports the fact that, in general, a form of attentional distraction - specifically, orthographic analysiswas the main strategy used to attempt to suppress the target words.

A speed-of-learning ANOVA was performed by comparing the amount of repetitions needed to learn the word pairs across each of the four main conditions. This analysis was crucial because of the possibility of a learning confound in which one condition was more overlearned than another. 
Specifically, negative pairs may have been learned more quickly than neutral pairs. The ANOVA showed no significant differences between the four conditions in learning rates. Furthermore, speed of learning was entered as a covariate in all ROI analyses to investigate the potential effects of this variable but did not result in significant differences from our original analyses (see the supplemental materials). This analysis suggests that learning rate was not a significant factor contributing to our results. This, however, does not rule out emotion's having an effect on memory during encoding. Previous research has shown that enhanced attention during the encoding of emotion items may account for short-term, but not long-term, gains in memory performance (Sharot \& Phelps, 2004). Therefore, it is possible that the differences seen in suppression between neutral and negative items in our results may have been due to enhanced encoding of emotional items over neutral items. Future studies manipulating attention at encoding, and using longer delays than those in the present study, could explicitly test this possibility.

\section{CONCLUSIONS}

The present study demonstrates that the emotionality of a target memory differentially modulates activity in the hippocampus and amygdala during attempts at suppressing recall. Suppression of negative target memories was associated with sustained activation of the hippocampus and a relative increase of amygdalar activation over time, relative to recall. Suppression of neutral target memories was associated with a relative decrease in hippocampal activation over time, relative to recall. The present findings of greater frontoparietal activation and lowered hippocampal activation during suppression of neutral words replicate those in Anderson et al. (2004). The putative control regions found during suppression in the present study, for both negative and neutral materials, are similar to those found in previous fMRI TNT studies that looked at neutral (Anderson et al., 2004) and negative (Depue et al., 2007) materials separately. Greater activation in the amygdala, left anterior insula, ACC, and left fusiform gyrus that might be related to continued activation in the hippocampus was found during attempts at suppressing negative words. Thus, for affective targets, similar control regions were engaged, but regions important for emotional and sensory processing (the amygdala, insula, ACC, fusiform gyrus) remained activated along with regions indexing explicit memory retrieval (the hippocampus). These regions are similar to those shown in Depue et al. (2007) to decrease in activity when emotional memories in the nothink condition are later forgotten. Discrepancies between the findings in the present study and those in Depue et al. (2007) in terms of hippocampus modulation during the suppression of negative stimuli should be considered in light of several crucial methodological differences. First, the present study used a high degree of learning not employed in previous studies. Second, unlike in the previous work, a specific strategy, attentional distraction through orthographic analysis, was given to the participants. Third, each repetition of suppression lasted $2 \mathrm{sec}$, whereas in the previous work, participants were given 4 sec during a suppression trial. Differences between negative and neutral pairs might have decreased if the participants had been given more time during each repetition to suppress recall. In other words, the findings are specific to attempts at suppression no longer than 2 sec. Fourth, this study included six repetitions of suppression, but previous work has usually included a larger amount of repetitions.

Overall, in the present study, when the same strategy was used, it seems as though, at least for word stimuli, the attempted suppression of arousing negative memories showed activity in several regions of the brain important for the representation of emotional memories. Using attentional distraction - specifically, orthographic analysis - to avoid recall of negative items may not engage the appropriate emotional control or regulatory systems. Exposure, along with forms of emotional regulation and reappraisal (Ochsner \& Gross, 2005), may, unlike attentional distraction, lead to decreased activation in these emotional areas. Direct comparisons of suppression strategies involving exposure, emotion regulation, and cognitive reappraisal will be important for future work.

\section{AUTHOR NOTE}

We thank the MRI techs, Thea Atwood and Rebecca Ward, for all of their assistance. We also thank the Lily Foundation for MetaCyte funding. Finally, we offer a sincere thanks to the anonymous reviewers for helpful comments on previous versions of the manuscript. Correspondence concerning this article should be addressed to A. J. Butler, Department of Psychological and Brain Sciences, Indiana University, 1101 East 10th St., Bloomington, IN 47405 (e-mail: butler7@umail.iu.edu).

\section{REFERENCES}

Abramowitz, J. S., Tolin, D. F., \& Street, G. P. (2001). Paradoxical effects of thought suppression: A meta-analysis of controlled studies. Clinical Psychology Review, 21, 683-703.

American Psychiatric Association (2000). Diagnostic and statistical manual of mental disorders (Rev. 4th ed.). Washington, DC: Author.

Anderson, M. C. (2001). Active forgetting: Evidence for functional inhibition as a source of memory failure. Journal of Aggression, Maltreatment \& Trauma, 4, 185-210.

Anderson, M. C., \& Green, C. (2001). Suppressing unwanted memories by executive control. Nature, 410, 366-369.

Anderson, M. C., \& Levy, B. J. (2009). Suppressing unwanted memories. Current Directions in Psychological Science, 18, 189-194.

Anderson, M. C., Ochsner, K. N., Kuhl, B., Cooper, J., RobertSON, E., Gabrieli, S. W., ET AL. (2004). Neural systems underlying the suppression of unwanted memories. Science, 303, 232-235.

Barnier, A. J., Conway, M. A., Mayoh, L., Speyer, J., Avizmil, O., \& HARRIS, C. B. (2007). Directed forgetting of recently recalled autobiographical memories. Journal of Experimental Psychology: General, 136, 301-322.

Beck, J. G., Gudmundsdottir, B., Palyo, S. A., Miller, L. M., \& Grant, D. M. (2006). Rebound effects following deliberate thought suppression: Does PTSD make a difference? Behavioral Therapy, 37, 170-180.

Bergstrom, Z. M., Velmans, M., Fockert, J., \& RichardsonKLAVEHN, A. (2007). ERP evidence for successful voluntary avoidance of conscious recollection. Brain Research, 1151, 119-133.

BJork, R. A., \& Woodward, A. E. (1973). Directed forgetting of individual words in free recall. Journal of Experimental Psychology, 99, 22-27.

Botvinick, M. M., Cohen, J. D., \& Carter, C. S. (2004). Conflict monitoring and anterior cingulate cortex: An update. Trends in Cognitive Sciences, 8, 539-546.

Bradley, M. M., \& Lang, P. J. (1999). Affective norms for English words (ANEW): Stimuli, instruction manual and affective ratings 
(Tech. Rep. C-1). Gainesville: University of Florida, Center for Research in Psychophysiology.

Brown, J. W., \& Braver, T. S. (2005). Learned predictions of error likelihood in the anterior cingulate cortex. Science, 307, 1118-1121.

Buchanan, T. W. (2007). Retrieval of emotional memories. Psychological Bulletin, 133, 761-779.

Bulevich, J. B., Roediger, H. L., III, Balota, D. A., \& Butler, A. C. (2006). Failures to find suppression of episodic memories in the think/ no-think paradigm. Memory \& Cognition, 34, 1569-1577.

Cabeza, R., Rao, S. M., Wagner, A. D., Mayer, A., \& Schacter, D. L. (2001). Can medial temporal lobe regions distinguish true from false? An event-related fMRI study of veridical and illusory recognition memory. Proceedings of the National Academy of Sciences, 98, 4805-4810.

Conway, M., \& Fthenaki, A. (2003). Disruption of inhibitory control of memory following lesions to the frontal and temporal lobes. Cortex, 39, 667-686.

Dalgleish, T., Hauer, B., \& Kuyken, W. (2008). The mental regulation of autobiographical recollection in the aftermath of trauma. Current Directions in Psychological Science, 17, 259-263.

DAlgleish, T., \& Yiend, J. (2006). The effects of suppressing a negative autobiographical memory on concurrent intrusions and subsequent autobiographical recall in dysphoria. Journal of Abnormal Psychology, 113, 467-473.

Depue, B. E., Banich, M. T., \& Curran, T. (2006). Suppression of emotional and non-emotional content in memory: Effects of repetition on cognitive control. Psychological Science, 17, 441-447.

Depue, B. E., Curran, T., \& Banich, M. T. (2007). Prefrontal regions orchestrate suppression of emotional memories via a two-phase process. Science, 317, 215-219.

Dolcos, F., LABAR, K. S., \& CABEZA, R. (2004). Interaction between the amygdala and the medial temporal lobe memory system predicts better memory for emotional events. Neuron, 42, 855-863.

Dunn, B. D., Billotti, D., Murphy, V., \& Dalgleish, T. (2009). The consequences of effortful emotion regulation when processing distressing material: A comparison of suppression and acceptance. Behaviour Research \& Therapy, 47, 761-773.

Duvernoy, H. M., Bourgouin, P., \& Vannson, J. L. (1999). The human brain: Surface, three-dimensional sectional anatomy with MRI, and blood supply. New York: Springer.

Ehlers, A., \& Clark, D. M. (2000). A cognitive model of posttraumatic stress disorder. Behaviour Research \& Therapy, 38, 319-345.

Eldridge, L. L., Knowlton, B. J., Furmanski, C. S., Bookheimer, S. Y., \& ENGEL, S. A. (2000). Remembering episodes: A selective role for the hippocampus during retrieval. Nature Neuroscience, $\mathbf{3}$, $1149-1152$

Fernández, G., Effern, A., Grunwald, T., Pezer, N., Lehnertz, K., DüMPELMANN, M., ET AL. (1999). Real-time tracking of memory formation in the human rhinal cortex and hippocampus. Science, $\mathbf{2 8 5}$, $1582-1585$

Fiez, J. A., \& Petersen, S. E. (1998). Neuroimaging studies of reading. Proceedings of the National Academy of Sciences, 95, 914-921.

Garavan, H., Ross, T. J., \& Stein, E. A. (1999). Right hemispheric dominance of inhibitory control: An event-related functional MRI study. Proceedings of the National Academy of Sciences, 96, 83018306.

Greenberg, D. L., Rice, H. J., Cooper, J. J., Cabeza, R., Rubin, D. C., $\&$ LABAR, K. S. (2005). Co-activation of the amygdala, hippocampus and inferior frontal gyrus during autobiographical memory retrieval. Neuropsychologia, 43, 659-674.

Hanslmayr, S., Leipold, P., Pastötter, B., \& BäUml, K.-H. (2009). Anticipatory signatures of voluntary memory suppression. Journal of Neuroscience, 29, 2742-2747.

Harvey, A. G., \& Bryant, R. A. (1998). The role of valence in attempted thought suppression. Behaviour Research \& Therapy, 36, 757-763.

Harvey, A. G., Bryant, R. A., \& Tarrier, N. (2003). Cognitive behaviour therapy for posttraumatic stress disorder. Clinical Psychology Review, 3, 501-522.

Hertel, P. T., \& Calcaterra, G. (2005). Intentional forgetting benefits from thought substitution. Psychonomic Bulletin \& Review, 12 , 484-489.

Hertel, P. T., \& Gerstle, M. (2003). Depressive deficits in forgetting. Psychological Science, 14, 573-578.
Joorman, J., Hertel, P. T., Brozovich, F., \& Gotlib, I. H. (2005). Remembering the good, forgetting the bad: Intentional forgetting of emotional material in depression. Journal of Abnormal Psychology, 114, 640-648.

Joseph, S., Dalgleish, T., Thrasher, S., Yule, W., Williams, R., \& HodgKinason, P. (1996). Chronic emotional processing in survivors of the Herald Free Enterprise disaster: The relationship of intrusion and avoidance at 3 years to distress at 5 years. Behaviour Research \& Therapy, 34, 357-360.

JosLYN, S. L., \& OAKeS, M. A. (2005). Directed forgetting of autobiographical events. Memory \& Cognition, 33, 577-587.

Kensinger, E. A., \& Schacter, D. L. (2005). Retrieving accurate and distorted memories: Neuroimaging evidence for effects of emotion. NeuroImage, 27, 167-177.

Khader, P., Burke, M., Bien, S., Ranganath, C., \& Rösler, R. (2005). Content-specific activation during associative long-term memory retrieval. NeuroImage, 27, 805-816.

LABAR, K. S., \& CABEZA, R. (2006). Cognitive neuroscience of emotional memory. Nature Reviews Neuroscience, 7, 54-64.

LEVy, B. J., \& ANDERSON, M. C. (2008). Individual differences in the suppression of unwanted memories: The executive deficit hypothesis. Acta Psychologica, 127, 623-635.

LitTRELL, J. (1998). Is the reexperience of painful emotion therapeutic? Clinical Psychology Review, 18, 71-102.

LUTCKe, H., \& FRAHM, J. (2008). Lateralized anterior cingulate function during error processing and conflict monitoring as revealed by highresolution fMRI. Cerebral Cortex, 18, 508-515.

Marx, B. P., Marshall, P. J., \& Castro, F. (2008). The moderating effects of stimulus valence and arousal on memory suppression. Emotion, 8, 199-207.

Mayou, R. A., Ehlers, A., \& Bryant, B. (2002). Posttraumatic stress disorder after motor vehicle accidents: 3-year follow-up of a prospective longitudinal study. Behaviour Research \& Therapy, 40, 665-675.

McCandliss, B. D., Cohen, L., \& Dehaene, S. (2003). The visual word form area: Expertise for reading in the fusiform gyrus. Trends in Cognitive Sciences, 7, 293-299.

Mecklinger, A., Parra, M., \& Waldhauser, G. T. (2009). ERP correlates of intentional forgetting. Brain Research, 1255, 132-147.

Miller, E. K. (2000). The prefrontal cortex and cognitive control. Nature Reviews Neuroscience, 1, 59-65.

Najmi, S., Wegner, D. M., \& Nock, M. K. (2007). Thought suppression and self-injurious thoughts and behaviors. Behaviour Research \& Therapy, 45, 1957-1965.

OCHSNER, K. N., \& Gross, J. J. (2005). The cognitive control of emotion. Trends in Cognitive Sciences, 9, 241-249.

Payne, B. K., \& Corrigan, E. (2007). Emotional constraints on intentional forgetting. Journal of Experimental Social Psychology, 43, 780-786.

Paz-Alonso, P. M., Ghetti, S., Matlen, B. J., Anderson, M. C., \& Bunge, S. A. (2009). Memory suppression is an active process that improves over childhood. Frontiers in Human Neuroscience, 3, 1-6.

Phan, K. L., Wager, T. D., Taylor, S. F., \& Liberzon, I. (2002). Functional neuroanatomy of emotion: A meta-analysis of emotion activation studies in PET and fMRI. NeuroImage, 16, 331-348.

Phelps, E. A. (2004). Human emotion and memory: Interactions of the amygdala and hippocampal complex. Current Opinion in Neurobiology, 14, 198-202.

Phelps, E. A., \& LeDoux, J. E. (2005). Contributions of the amygdala to emotion processing: From animal models to human behavior. $\mathrm{Neu}$ ron, 48, 175-187.

Phelps, E. A., \& Sharot, T. (2008). How (and why) emotion enhances the subjective sense of recollection. Current Directions in Psychological Science, 17, 147-152.

Pruessner, J. C., Li, L. M., Serles, W., Pruessner, M., Collins, D. L., Kabani, N., ET AL. (2000). Volumetry of hippocampus and amygdala with high-resolution MRI and three-dimensional analysis software: Minimizing the discrepancies between laboratories. Cerebral Cortex, 10, 433-442.

Purdon, C. (1999). Thought suppression and psychopathology. Behaviour Research \& Therapy, 37, 1029-1054.

Ramautar, J. R., Slagterb, H. A., Koka, A., \& Ridderinkhof, K. R. (2006). Probability effects in the stop-signal paradigm: The insula and the significance of failed inhibition. Brain Research, 1105, 143-154. 
Rassin, E., Merckelbach, H., \& Muris, P. (2000). Paradoxical and less paradoxical effects of thought suppression: A critical review. Clinical Psychology Review, 200, 975-995.

Reiman, E. M., Lane, R. D., Ahern, G. L., Schwartz, G. E., DavidSON, R. J., FRISTON, K. J., ET AL. (1997). Neuroanatomical correlates of externally and internally generated human emotion. American Journal of Psychiatry, 154, 918-925.

Sharot, T., Martorella, E. A., Delgado, M. R., \& Phelps, E. A. (2007). How personal experience modulates the neural circuitry of memories of September 11. Proceedings of the National Academy of Sciences, 104, 389-394.

Sharot, T., \& Phelps, E. A. (2004). How emotional arousal modulates memory: Disentangling the effects of attention and retention. Cognitive, Affective, \& Behavioral Neuroscience, 4, 294-306.

Shipherd, J. C., \& Beck, J. G. (1999). The effects of suppressing trauma-related thoughts on women with rape-related posttraumatic stress disorder. Behaviour Research \& Therapy, 37, 99-112.

SlotNick, S. D. (2004). Visual memory and visual perception recruit common neural substrates. Behavioral \& Cognitive Neuroscience Reviews, 3, 207-221.

TAlAiRACH, J., \& TOURNOUX, P. (1988). A co-planar stereotaxic atlas of the human brain: 3-dimensional proportional system. An approach to cerebral mapping (M. Rayport, Trans.). New York: Thieme.

Vaidya, C. J., Zhao, M., Desmond, J. E., \& Gabrieli, J. D. E. (2002). Evidence for cortical encoding specificity in episodic memory:
Memory-induced re-activation of picture processing areas. Neuropsychologia, 40, 2136-2143.

Watanabe, J., Sugiura, M., Kawashima, R., Sato, K., Maeda, Y., SATO, Y., ET AL. (2002). The human prefrontal and parietal association cortices are involved in NO-GO performances: An event-related fMRI study. NeuroImage, 17, 1207-1216.

Wegner, D. M., Shortt, J. W., Blake, A. W., \& Page, M. S. (1990). The suppression of exciting thoughts. Journal of Personality \& Social Psychology, 58, 409-418.

Wessel, I., Wetzels, S., Jelicic, M., \& Merckelbach, H. (2005). Dissociation and memory suppression: A comparison of high and low dissociative individuals' performance on the think-no think task. Personality \& Individual Differences, 39, 1461-1470.

Wheeler, M. E., Petersen, S. E., \& Buckner, R. L. (2000). Memory's echo: Vivid recollection activates modality-specific cortex. Proceedings of the National Academy of Sciences, 97, 1125-1129.

\section{SUPPLEMENTAL MATERIALS}

The individual speed-of-learning results for our participants and alternate SPM and ROI analyses may be downloaded from http://cabn .psychonomic-journals.org/content/supplemental.

(Manuscript received April 27, 2009; revision accepted for publication November 11, 2009.) 